\title{
A Novel Robot with a Toroidal Workspace for Coring Applications
}

\author{
Dave Hill \\ Centre for Additive Manufacturing \\ School of Engineering \\ RMIT University \\ Melbourne, Australia \\ dave.hill@rmit.edu.au \\ Martin Leary \\ Centre for Additive Manufacturing \\ School of Engineering \\ RMIT University \\ Melbourne, Australia \\ martin.leary@rmit.edu.au
}

\author{
Tom Williamson \\ Centre for Additive Manufacturing \\ School of Engineering \\ RMIT University \\ Melbourne, Australia \\ tom.williamson@rmit.edu.au \\ Milan Brandt \\ Centre for Additive Manufacturing \\ School of Engineering \\ RMIT University \\ Melbourne, Australia \\ milan.brandt@rmit.edu.au
}

\author{
Chow Yin Lai \\ Department of Electronic and Electical \\ Engineering \\ University College London \\ London, England \\ c.lai@ucl.ac.uk \\ Peter Choong \\ University of Melbourne \\ Department of Surgery \\ Department of Orthopaedics, \\ St. Vincent's Hospital Melbourne \\ Melbourne, Australia \\ pchoong@unimelb.edu.au
}

\begin{abstract}
Removing a volume of tissue as a single mass can be a complicated operation, and in many cases it would be advantageous to be able to remove more complex geometries and preserve nearby critical regions. In this paper, we present a novel robot with 2 degrees of freedom, designed for cutting around a parametric torus-shaped volume. The kinematics of this system make it possible to drive the tip of the linkage such that it does not collide with any point not on the surface of the specified torus. Two types of tool paths can be followed, with the torus geometry defined to fit a target region of material. Three bone tumor cases were used for simulating the approach. Compared to more standard tri-planar cutting approaches, a toroidal resection could improve upon the amount of healthy bone resected, and reduce surgical access requirements.
\end{abstract}

Keywords - surgical robotics, elaborate resection, torus

\section{INTRODUCTION}

In the field of orthopaedic oncology, bone tumours must be removed en-bloc (in one piece) with a cuff of healthy tissue, to ensure no diseased tissue remains in the patient [1]. In a clinical setting, removing a volume of material without intersecting the target volume itself is a complicated procedure. Tumor resections are typically performed with planar cuts, precisely aligned with anatomical features or the axes of the bone, to separate diseased tissue from the surrounding healthy bone. While a planar cut set at an adequate distance from a tumor increases the likelihood of complete removal of the diseased tissue [2], this may also result in resecting critical anatomy, or more healthy tissue than is necessary. More complex non-planar cutting trajectories can be performed with high-speed burrs, which can be directed using patient-specific instruments [3]. This allows for resections which more closely conform to the tumor surface, where the volume of collaterally resected healthy bone is minimized.

Complex orthopaedic hardware can allow alternative resection volumes to be generated. In hip arthroplasty revision surgery, spherical acetabular cups can be removed with specialised tooling such as Stryker's EZout or Zimmer's Explant System [4]. These devices allow a hemispherical volume to be removed without intersecting the volume; the cutting tool follows itself through its cutting path, remaining on the surface of the sphere, conforming to the desired shape and minimizing the volume of healthy bone that is removed with the implant.

In many surgical cases it would be advantageous to extend this principle of resection waste minimization to more complex or alternative (non-spherical) shapes. Tool paths for these shapes could, for example, allow a surgeon to preserve anatomy (such as ligament attachment points) by cutting behind the critical region, reduce the volume of tissue taken with the tumour, or make an orthopaedic reconstruction less complex. The removal of such volumes requires the development of surgical tools that can follow itself through a path or remain exclusively on the surface of a shape.

A number of groups working on flexible and soft robotics have developed systems in which a tool fits through a narrow irregular path. Continuum-type robots can conform to complex geometries, such as tori or conics. While the flexibility of these systems theoretically allow indirect access to a volume, their wide range of poses comes from their increased flexibility, a property which does not lend itself to high rigidity applications such as accurately cutting bone [5]. However, the approach has proven feasible for drilling of curved trajectories [6].

Between the two extremes of constant positive curvature (sphere) and generic freely defined curved surfaces lies the torus. A torus is a ring-shaped three dimensional (3D) surface of revolution, produced by revolving a circle about an axis coplanar to the circle. It can be defined based on two radii and has a number of properties that make it an interesting candidate as a bone removal volume, not least that the multiple radii may allow the volume to more closely conform to the tumour surface.

Studies on Bricard linkages touch on a toroid-based tool paths, specifically an RR dyad capable of generating a singular toroid [7]. While the proposed RR dyad design could follow a standard torus-shaped tool path, the linkage would not be suitable for en-bloc resections, or coring applications, as components of the design would be required to pass through the toroid volume.

In this paper, we present a novel robot kinematics with 2 degrees of freedom, with a kinematic trajectory that lies entirely on a torus. With an appropriate cutting tool fixed at the end of the linkage, this may allow for a 
reduction in loss of healthy bone in en-bloc tumor resection surgery, and potentially prevent removal of otherwise unavoidable critical structures. More generally, we hypothesize that it is possible to design a robotic system that is capable of performing a coring action 'around a corner'. Due to the kinematics of this system it will be possible to position the end effector such that it does not collide with any point not on the surface of the specified torus.

Despite extensive review of modern and historic scholarly literature, patents, and commercial devices, to our knowledge this concept has not been previously explored.

Within this research we describe a torus-type robot in detail. Section II of this paper outlines the proposed robot design, along with the derivation of the kinematics for a robot with a toroidal workspace, and two methods of planning parametric tool paths. In section III, the concept is demonstrated through simulated planning on three bone tumour cases, including measurements of collaterally resected healthy bone (resection waste), compared to a more standard planar resection. Finally in section IV the advantages and disadvantages of a torus-type robot are discussed, as well as the necessary future work towards the realization of a functional robotic system capable of toroidal resection.

\section{MATERiAls \& MethoD}

A torus is a ring-shaped three dimensional (3D) surface of revolution, produced by revolving a circle about an axis coplanar to the circle. The torus minor radius $\left(R_{2}\right)$ is the radius of the revolved circle, and the major radius $\left(R_{1}\right)$ is the distance between the central axis and the centre of the revolved circle. A torus is typically aligned in Cartesian coordinates such that the central axis is coincident with the $\mathrm{z}$ axis, with the $\mathrm{z}$ plane defined as the major plane of the torus (Fig. 1). An alternative method of generating a torus is by specifying an outer diameter (OD), (equivalent to $2 \times\left(R_{1}+R_{2}\right)$ ), and an inner diameter (ID) (equivalent to $2 \times\left(R_{1}-R_{2}\right)$ ). A torus with $R_{2}>R_{1}(I D<0)$ will self-intersect, and is referred to as a spindle torus, while those with $R_{2}<R_{1}(I D>0)$ are referred to as a ring torus

A torus' surface has positive, negative, and zero Gaussian curvature. Viewing a torus parallel to the central or $\mathrm{z}$ axis, all of the surface less than the major radius possesses negative Gaussian curvature, while all of the surface greater than the major radius possesses positive Gaussian curvature. Finally, the regions directly above and below the major radius have zero Gaussian curvature.

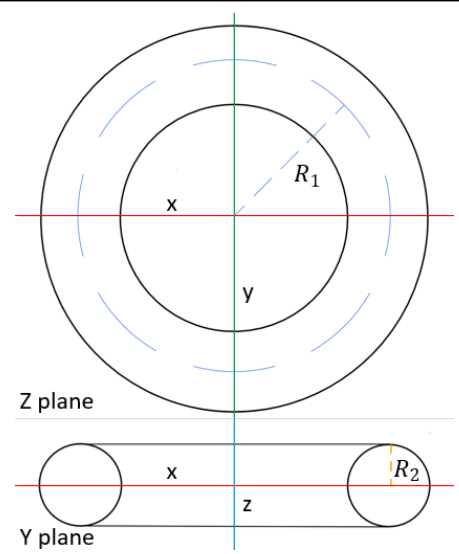

Fig. 1. Orthogonal views of a standard torus, where $R_{1}$ is the major radius and $R_{2}$ is the minor radius

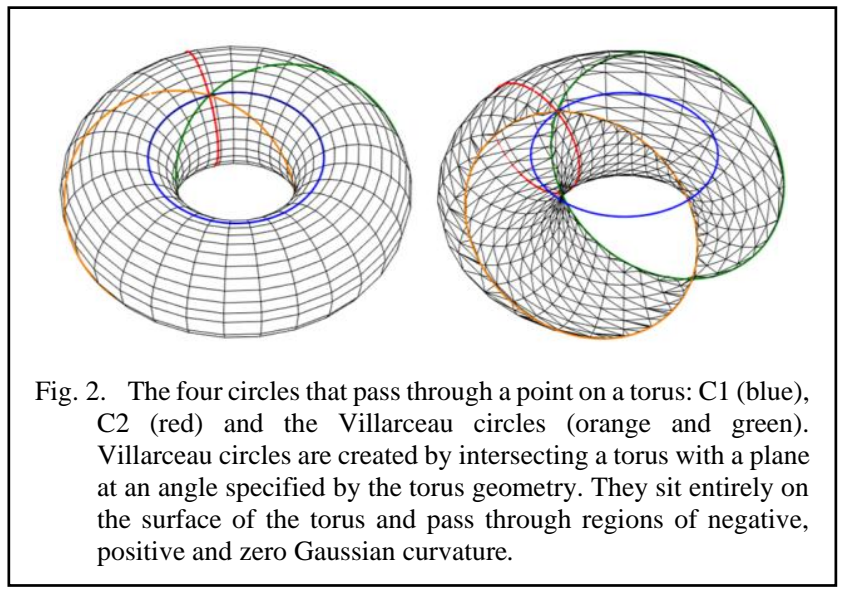

\section{A. Description of the torus-type robot}

Any point on a ring torus surface can have four circles pass through it, all of which are in full contact with the surface. The first circle $(\mathrm{C} 1)$ lies in the plane perpendicular to the torus central axis. The second circle (C2) lies in the plane parallel to the torus central axis, and is equivalent to the circle revolved about the central axis to produce the surface. The third and fourth circles, known as Villarceau Circles (VC) [8], are found in an oblique plane passing through the selected point and the centroid of the torus, at an angle tangent to the torus surface, (equivalent to the inverse sine of $R_{2}$ divided by $R_{1}$ ). The radius of a VC is fixed to the torus, and is equal to $R_{1}$. The VC centre point lies on a circle with radius $R_{2}$, in the plane perpendicular to the torus central axis, passing through the torus centre point. Each of these circles is shown in Fig. 2.

For any given point on a ring torus, circle $\mathrm{C} 1$ will pass through only one of the Gaussian curvatures (positive, negative, or zero) of the torus, while circle $\mathrm{C} 2$ and both VC will contact all three. Additionally, the radii of $\mathrm{C} 2$ and both $\mathrm{VC}$ are determined by the major and minor radii of the torus, while the radius of $\mathrm{C} 1$ varies with the position of the point. Finally, while the centre point of $\mathrm{C} 1$ lies on the torus central axis and the centre point of $\mathrm{C} 2$ lies within the torus volume, the centre points of the $\mathrm{VC}$ lie within the hole of the torus. In contrast to $\mathrm{C} 1$ and $\mathrm{C} 2$, a $\mathrm{VC}$ can have both a fixed radius and a centre point outside the torus surface, and still reach every point of the surface of the torus.

Using these features as a basis for the torus-type robot kinematics, a VC-based design allows for a robot of fixed kinematic length and dimensions to access all points of a torus surface, and accommodate for the positive, negative, and zero Gaussian curvature. In the following section we describe the kinematics of a torus-type robot based on these Villarceau circles.

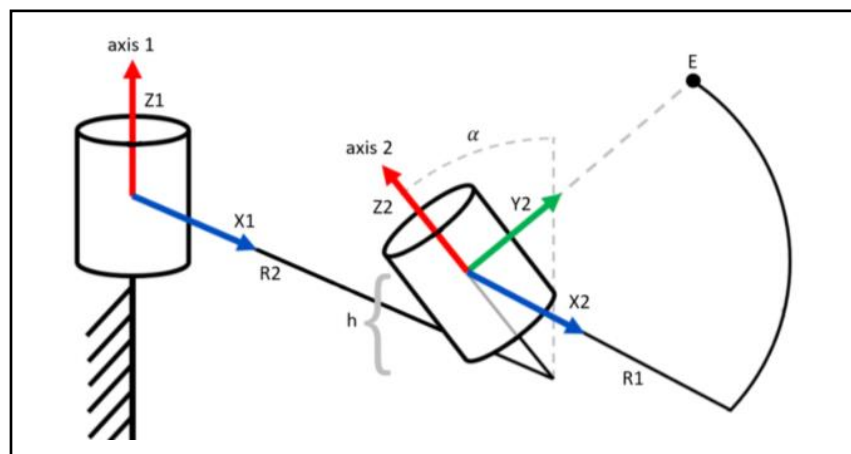

Fig. 3. Simplified DH representation of proposed robot. 


\section{B. Derivation of Robot Kinematics}

A VC based robot can be constructed from a base and two links connected by two rotational joints (Fig. 3), defined by the Denavit-Hartenburg (DH) parameters shown in Table I.

TABLE I. LINKAGE DH PARAMETERS

\begin{tabular}{|c|c|c|c|c|}
\hline $\boldsymbol{i}$ & $\boldsymbol{a}_{\boldsymbol{i}-\mathbf{1}}$ & $\boldsymbol{\alpha}_{\boldsymbol{i}-\mathbf{1}}$ & $\boldsymbol{d}_{\boldsymbol{i}}$ & $\boldsymbol{\theta}_{\boldsymbol{i}}$ \\
\hline 1 & 0 & 0 & 0 & $\theta_{1}$ \\
\hline 2 & $R_{2}$ & $\sin ^{-1} \frac{R_{2}}{R_{1}}=\alpha$ & $h$ & $\theta_{2}$ \\
\hline
\end{tabular}

The length of the first link is equal to $R_{2}$, and the length of the second link is equal to $R_{1}$. The change in position along the $\mathrm{z}$ axis of the previous joint is defined by free variable $h$, and the twist angle of the second joint about the previous $x$ axis is $\alpha$. The forward kinematics of this structure can then be derived, with a final transformation between the centre of the torus and tip of the end effector given by (1), where:

$$
{ }^{0} P_{E}=\left[\begin{array}{c}
-R_{1} \cos \theta_{1} \sin \theta_{2}-R_{1} \sin \theta_{1} \cos \alpha \cos \theta_{2} \\
+R_{2} \cos \theta_{1}+\sin \theta_{1} \sin \alpha h \\
\frac{-R_{1} \sin \theta_{1} \sin \theta_{2}+R_{1} \cos \theta_{1} \cos \alpha \cos \theta_{2}}{+R_{2} \sin \theta_{1}-\cos \theta_{1} \sin \alpha h} \\
R_{1} \sin \alpha \cos \theta_{2}+\cos \alpha h
\end{array}\right]
$$

The inverse kinematics of the robot are given by (2) to (5), where:

$$
\begin{gathered}
\theta_{1}=\operatorname{atan} 2(K 2, K 1)-\operatorname{atan} 2(-y, x) \\
\theta_{2}=\cos ^{-1} \frac{z-\cos \alpha h}{R_{1} \sin \alpha}
\end{gathered}
$$

where $\mathrm{x}, \mathrm{y}$, and $\mathrm{z}$ are coordinates of tool path points, and

$$
\begin{gathered}
K 1:=\left(-R_{1} \sin \theta_{2}+R_{2}\right) \\
K 2:=\left(-R_{1} \cos \alpha \cos \theta_{2}+h \sin \alpha\right)
\end{gathered}
$$

Thus, given any point on the surface of the torus, a pair of unique joint angles can be recovered. The defined structure of

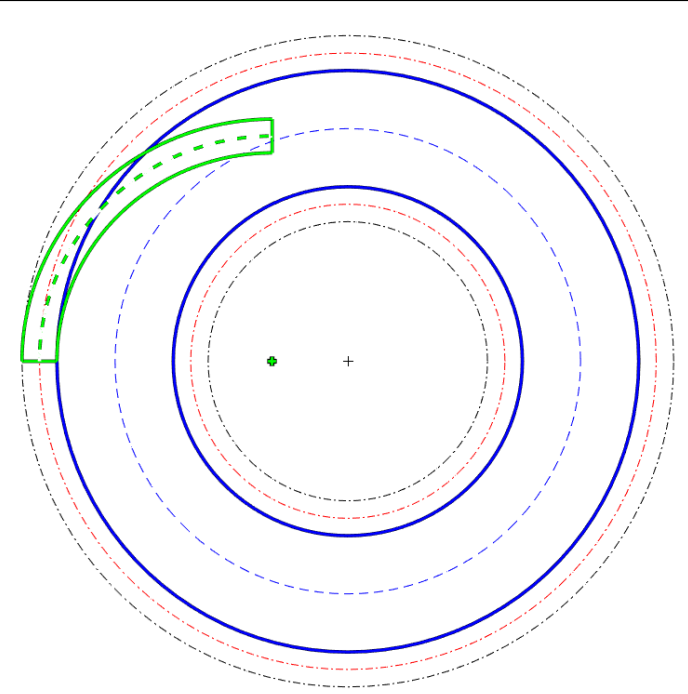

Fig. 4. Removal of blue torus with green tool is performed by resecting the torus with red dashed lines. After resection, resulting void will be equivalent to black dashed line. the system ensures that it will not interfere with the internal torus volume.

Note that an infinitely thin end effector is assumed with the system as defined above. In reality, when calculating the kinematics and generating the tool path, the torus minor radius will increase by the radius of the robot end effector (cutting tool), while the resulting torus void has a minor radius equal to the excision minor radius plus the end effector diameter (Fig. 4). The forward and inverse kinematics for a generic VC based robot were implemented in Matlab 2018a (MATLAB, 2018, 9.4.0.813654 (R2018a). The MathWorks Inc).

\section{Path Planning}

Path planning is performed in three stages. First, a generic path is created covering the complete surface of the defined torus. This path is then refined by removing points that are not required for removal of bone volume (i.e. points on the path that are not within the bone). Finally, the path points are ordered to prevent sudden discontinuities in position and ensure smooth motion of the robot. Two kinematic approaches were investigated for the linkage to follow: a toroidal helix, and discrete loops (Fig. 5).

The toroidal helix tool path is generated by (6) to (8), where:

$$
\begin{gathered}
x=\left(R_{1}+\cos (n \theta) \cdot R_{2}\right) \cos (\theta) \\
y=\left(R_{1}+\cos (n \theta) \cdot R_{2}\right) \sin (\theta) \\
z=\sin (n \theta) \cdot R_{2}
\end{gathered}
$$

where $n$ is the number of winds around a torus of major radius $R_{1}$ and minor radius $R_{2}$. This results in a helical tool path wrapped around a torus, with $n$ winds. This approach sorts the points in correct order upon generation, thereby positioning the tool path within the bone and eliminating unnecessary points leads to an ordered tool path with no additional sorting required.

The second tool path approach is comprised of discrete loops revolved around a central axis, and is generated by (9) to (11), where:

$$
\begin{gathered}
x=\left(R_{1}+\cos (\theta) \cdot R_{2}\right) \cos (\varphi) \\
y=\left(R_{1}+\cos (\theta) \cdot R_{2}\right) \sin (\varphi) \\
z=\sin (\theta) \cdot R_{2}
\end{gathered}
$$

where $\theta$ is equal to partitions at the perimeter of the torus, and $\varphi$ is partitions along the azimuth of the torus. In essence, the previously described $\mathrm{C} 2$ circle is generated with points at $\theta$,

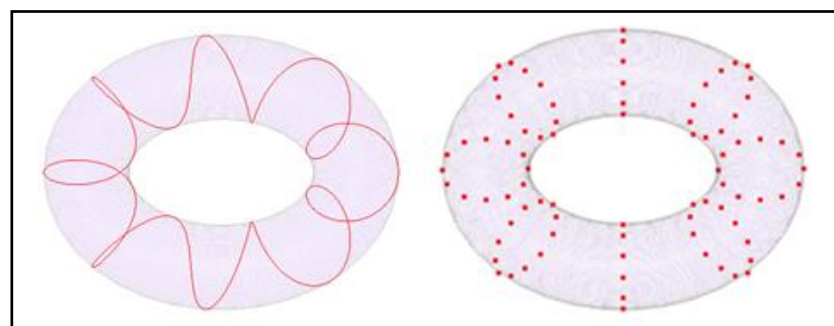

Fig. 5. Helical tool path using $n=8$ (left) and loop tool path using $\theta=12$ and $\varphi=8$ (right) 

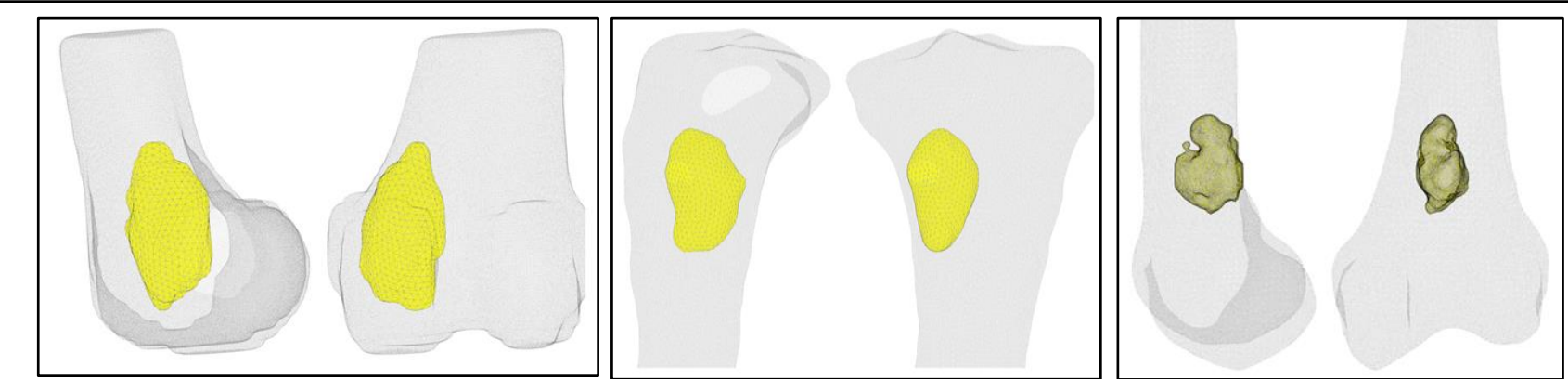

Fig. 6. Coronal and sagittal views of segmented bone (grey) and tumor (yellow) cases

then revolved about the central axis of the torus at steps of $\varphi$. The points comprising each circle at $\varphi$ are ordered using $\operatorname{atan} 2\left(\theta_{y}, \theta_{\mathrm{x}}\right)$.

\section{Torus Generation}

While the proposed approaches can be applied to a torus with arbitrary values for $R_{1}$ and $R_{2}$, disproportionate values may result in impractical tool design. Allowing any values for $R_{1}$ and $R_{2}$ may produce a self-intersecting spindle torus, a torus with $R_{2}$ equal to zero or infinite (equivalent to a sphere and cylinder respectively), or a torus with excessively large disparities between the $R_{1}$ and $R_{2}$ link lengths. In order to ensure the torus fully encloses the tumor, and the robot link lengths are sized proportional to a small surgical robot, the values for $R_{1}$ and $R_{2}$ are generated based on the tumor geometry.

First, the tumor centroid is determined based on the average value of the points comprising the surface mesh (acquisition described in the following section). Then, the tumor and bone are translated to the tumor centroid and rotated about the 3D coordinate space. The surface mesh points are projected in 2D on the y plane, and the most-distant surface mesh point from the tumor centroid is set as $R_{2}$.

After defining the value for $R_{2}$, the value for $R_{1}$ is set as two times $R_{2}$ with provision to adjust $R_{1}$ based on an arbitrary scaling value. Additionally, a scaling value can be applied to $R_{2}$ to ensure elongated or irregularly-shaped tumors are fully enclosed within the torus bounds. Defining the torus in this manner ensures generation of ring tori only.

\section{E. Proof of concept and demonstration}

Three example tumour cases were utilized for evaluation and demonstration of the torus-type robot (Fig. 6). These cases were based on real patient data, acquired with permission from the local ethical review board (approval ID:
LNR/18/SVHM/21). Anonymized CT and MRI data were acquired, the bone and tumour segmented using open source medical imaging software (MITK, DKFZ, Germany) and exported as mesh files. The surface meshes of both the bone and tumor were imported into Matlab, and converted to 3D volumetric arrays of uniformly spaced $2 \mathrm{~mm}$ cubic voxels, whereby voxel positions outside of the bone were discarded. For each case, a torus was manually positioned to fully enclose the tumour, while also attempting to minimize the number of voxels of bone included in the resection. Cutting paths were generated using both the helix and loop methods and the joint angles calculated.

Finally, simple robotic links representing the kinematic structure of the robot were designed (Solidworks, Dassault Systems, France) and exported as meshes. Visual representations of the final robot geometry were automatically generated, with the vertices comprising the linkage meshes scaled to fit the proposed torus geometry. Total path lengths using both path generation methods were assessed, in addition to the total volume of tissue removed by the specified torus. The resection waste was reported as a percentage of healthy bone, counting the number of bone voxels within the resection, compared to the volume of the entire resection (tumor voxels plus bone voxels). As a comparison, a tri-planar resection was also manually planned for each case, with cutting planes set proximal and distal to the tumor, with the final plane positioned perpendicular to the previous cuts.

\section{RESULTS}

The generated robot design and one of the tool paths are shown in Fig.7, with tool path values of $\theta=50$ and $\varphi=40$. The volume of healthy bone removed by a toroidal cut for each tumour case, and the comparative three plane cut are shown in Fig. 8 and Table II.

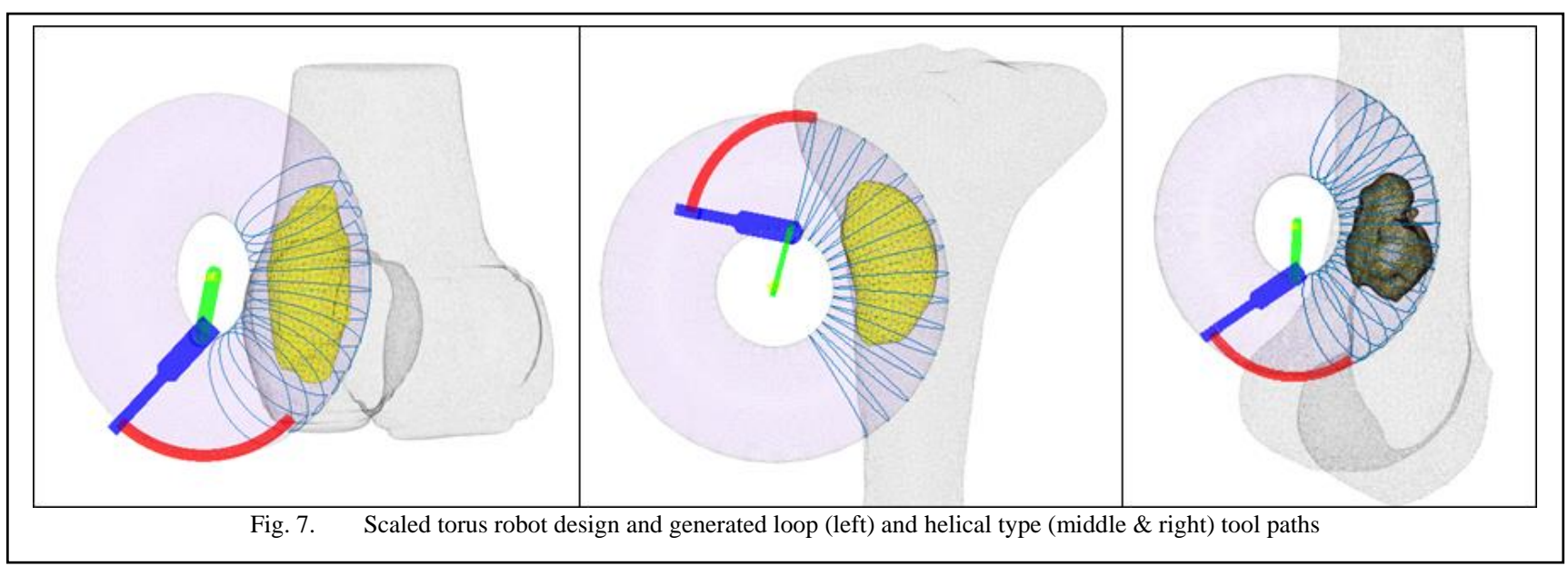




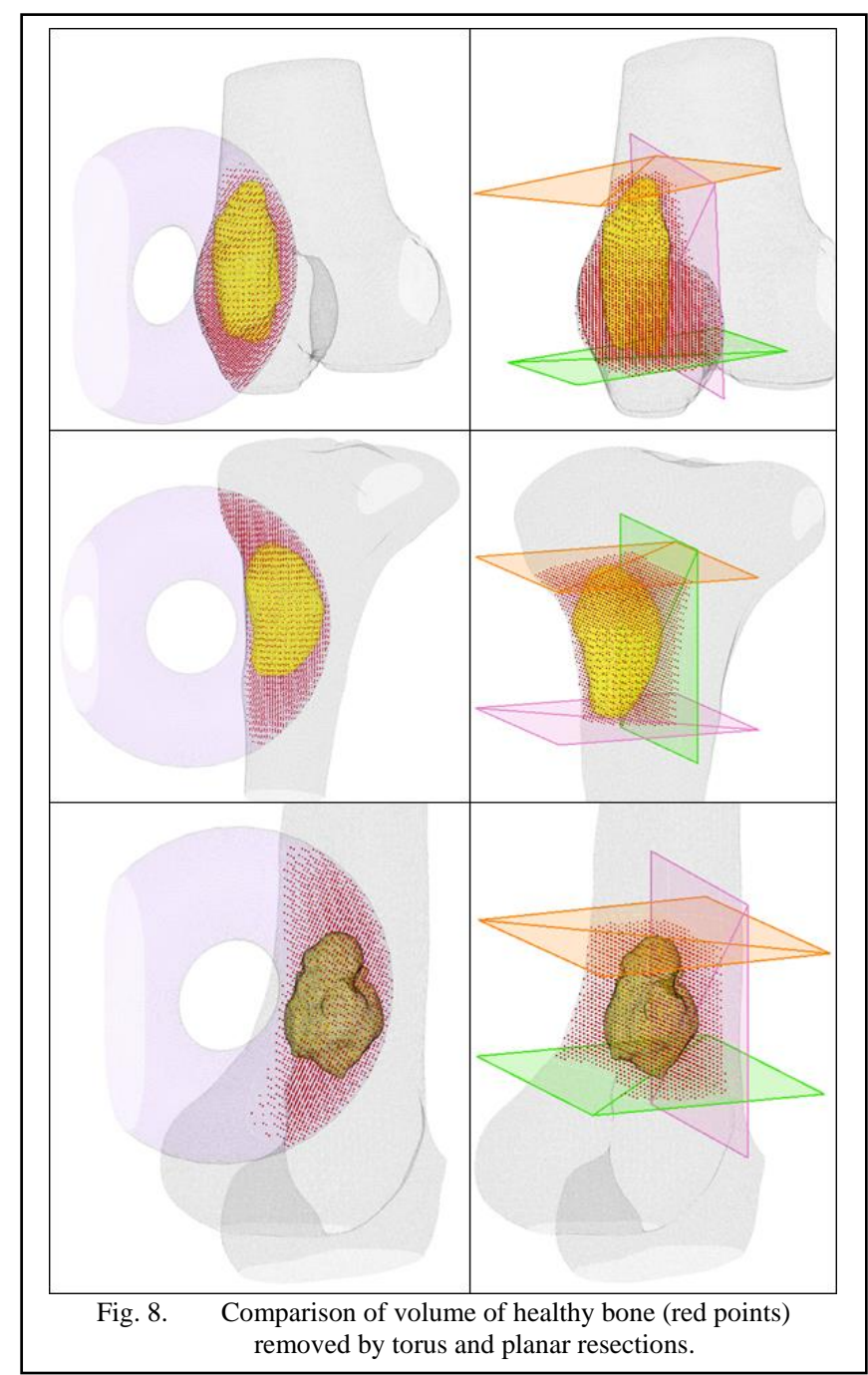

TABLE II. TORUS PARAMETERS, PATH PENGTH, AND VOLUME REMOVED

\begin{tabular}{|c|c|c|c|}
\hline R1 (mm) & Case 1 & Case 2 & Case 3 \\
\hline R2 (mm) & 33.180 & 30.269 & 26.011 \\
\hline $\begin{array}{c}\text { Helical path length } \\
\text { (mm) }\end{array}$ & 1005 & 1444 & 1232 \\
\hline $\begin{array}{c}\text { Loop path length } \\
\text { (mm) }\end{array}$ & 1064 & 1582 & 1356 \\
\hline $\begin{array}{c}\text { Torus resection } \\
\text { volume } \\
\text { (inc. tumor) (mm }{ }^{3} \text { ) }\end{array}$ & 30230 & 36860 & 23110 \\
\hline $\begin{array}{c}\text { Resection waste } \\
\text { (\% healthy bone) }\end{array}$ & $59.59 \%$ & $66.84 \%$ & $77.88 \%$ \\
\hline $\begin{array}{c}3-\text { planar cut } \\
\text { resection volume } \\
\text { (inc. tumor) (mm }{ }^{3} \text { ) }\end{array}$ & 53280 & 37560 & 28544 \\
\hline $\begin{array}{c}\text { 3-planar cut } \\
\text { resection waste } \\
\text { (\% healthy bone) }\end{array}$ & $76.60 \%$ & $67.45 \%$ & $82.09 \%$ \\
\hline
\end{tabular}

Table II shows the calculated robot parameters of the generated robots, the difference in tool path length, and the difference in resection waste between the torus cut and a manually planned three plane cut.

The resection waste is calculated as a percentage of the number of bone voxels in the resection compared to the number of tumor voxels within the bone and the resection.
Thus, a perfectly conformal cut that removes only tumor and no collateral bone has no waste, while a resection with a waste of $100 \%$ removes only healthy bone. For case 2 , the torus cut had a comparable resection waste to that produced by the planar cut ( $<1 \%$ difference), while case 3 torus resection had a marginal improvement to resection waste (4\% difference) over the planar cut. For the case 1 torus resection, there was an appreciable improvement in resection waste $(16 \%$ difference) to the planar cut approach.

\section{DISCUSSION}

This work has described a novel robot kinematics with 2 degrees of freedom, designed for cutting around a parametric torus-shaped volume. The kinematics of this system make it possible to drive the tip of the linkage such that it does not collide with any point not on the surface of a specified torus. Initial simulation results demonstrate the ability of the robot to remain on a toroidal surface with the variation of only the joint angles, and without changing link lengths or other kinematic parameters. Also demonstrated was the ability of the design to follow both helical and stepped tool paths, and the potential for sparing of healthy bone or avoidance of critical structures during bone tumour surgery.

The VC tool design has a number of potential advantages in orthopaedic surgical applications. The ability to cut underneath critical tissue is realized in the examples shown, removing a curved core of material including a tumor and avoiding areas of ligament attachment. The resulting void could then be filled with a printed implant, or bone cement. As a geometry to fill, a torus has an advantage that there would be only one rotational degree of freedom for a fixed implant, such that a printed implant would to some extent exhibit inherent mechanical fixation. While not easily measurable, the surgical access required to remove the diseased tissue could potentially be reduced by using the torus resection, compared to more standard planar cuts. This may have the additional benefit to the patient of a surgery being less invasive or traumatic, while the reduced wound size would be less susceptible to complications such as bleeding, pain and infection. Moreover, smaller surgery may also enable earlier return of limb function. Furthermore, the design of the robot is modular and miniature, allowing it to be attached to the end of a larger robotic arm system. In the scheme of robotic surgical applications, connecting the $\mathrm{VC}$ torus tool to a larger robotic arm would mean any motion of the patient could be accounted for, allowing the torus robot to perform the resection and the larger system to only control tool position relative to the patient. Additionally, in the current robot design, the final link is a $90^{\circ}$ arc, limiting the cutting depth to $90^{\circ}$ from a single direction. In cases where the full length of the cut exceeds the length of the tool, this can be addressed by cutting halfway, retracting the tool, turning the robot around, and re-entering the cut from the other end of the torus.

The definition of the torus sizes was performed manually for each of the trials based on the tumour shape and with a ratio of 2 between $R_{1}$ and $R_{2}$. For case 1 this ratio was increased to 2.25 , as the length of the tumor resulted in a nonenclosing torus with a ratio of 2 . The tumours in these examples were all of similar size, and as such it would be possible to perform all three cases with a single tool of size $R_{1}$ of $34 \mathrm{~mm}$ and $R_{2}$ of $16 \mathrm{~mm}$. From a practical standpoint, having set tool sizes and linkages would increase the manufacturability of the robot, although restricting the variability of $R_{1}$ would increase the resection waste of a given 
cut. Furthermore, it is unlikely that all tumours would fit within this torus, and thus a set of robots with multiple sizes (equivalent to small, medium, and large), a single robot with modifiable link lengths and the custom manufacturing of case specific robots are possibilities for managing more variable tumour sizes. It is likely the CAD model used in the simulations is an over-simplification of the design required to perform a toroidal resection with a physical prototype. Detailed designs and prototyping will be the subject of future work.

The primary challenge of this robot is the type of tool required to cut or ablate material. A rotary burr type tool faces some engineering challenges, including miniaturization, stiffness, and potential cutting inefficiencies at certain positions. Some of these challenges could be addressed using a contactless cutting technology such as a laser fiber optic cutting approach. By positioning the tip of the fiber optic close to the tool path, then gradually advancing the tip of the fibre optic into the resulting ablation crater. A fibre optic would also minimize the volume of bone lost to the tool itself, allowing for resections with less collateral healthy bone. Additional challenges exist with ensuring there is sufficient space between the workspace, and the second joint. As the size of the torus cut is determined by the length of the links, there may be instances where excessive material above the cutting site would prevent the full range of motion required by the torus robot to perform the cut. Positioning the robot such that the robot centre is not above the patient would minimize the likelihood of interference. Additionally, it may be possible to negate this issue to an extent with the addition of a larger robotic arm system as previously discussed. One drawback of this cutting approach is that the torus cannot terminate the cut midway through the resection. For a cut to terminate prior to passing through the entire volume, a blind oblique 'facing' cut would need to be performed, parallel to the torus $\mathrm{z}$ axis, aligned with a previously mentioned $\mathrm{C} 2$ circle. This facing cut could save additional bone, but would require a change in tool.

Future work will focus on two major areas: first, the development of physical prototypes and miniaturized cutting approaches. Second, methods for optimization of the torus size and position will be investigated. These optimization methods will focus on the ability of the approach to minimize bone loss in comparison to a range of existing and novel cutting geometries.

\section{CONCLUSION}

This work has presented a novel robotic architecture capable of reaching behind surfaces. The system is designed such that its workspace is constrained to the surface of a torus, allowing the intact resection of a toroidal volume. The system kinematics were derived and described and two methods for planning of toroidal paths described. Examples were shown within the context of removal of bone tumours. It was shown that in certain cases, the toroidal resection plan minimises the removal of healthy bone compared to more traditional planar resections.

\section{ACKNOWLEDGMENT}

The authors acknowledge the facilities, and the scientific and technical assistance of the RMIT Advanced Manufacturing Precinct.

\section{REFERENCES}

[1] T. J. C. Pazionis, H. Alradwan, B. M. Deheshi, R. Turcotte, F. Farrokhyar, and M. Ghert, "A systematic review and metaanalysis of En-Bloc vs Intralesional Resection for Giant Cell Tumor of Bone of the Distal Radius.," Open Orthop. J., vol. 7 , pp. 103-108, 2013.

[2] L. Jeys, G. Morris, S. Evans, J. Stevenson, M. Parry, and J. Gregory, "Surgical Innovation in Sarcoma Surgery," Clin. Oncol., vol. 29, no. 8, pp. 489-499, 2017.

[3] D. A. Müller, Y. Stutz, L. Vlachopoulos, M. Farshad, and P. Fürnstahl, "The accuracy of three-dimensional planned bone tumor resection using patient-specific instrument," Cancer Manag. Res., vol. 12, pp. 6533-6540, 2020.

[4] L. Kwong, F. Billi, S. Keller, A. Kavanaugh, A. Luu, and W Paprosky, "A Comparative Study between the Stryker EZ out Powered Acetabular Revision System and the Zimmer Explant Acetabular Cup Removal Systems," Tech. Orthop., vol. 00, no. 00, pp. 1-8, 2019.

[5] M. Runciman, A. Darzi, and G. P. Mylonas, "Soft Robotics in Minimally Invasive Surgery," Soft Robot., vol. 6, no. 4, pp. 423443, 2019.

[6] A. Sendrowicz, M. Scali, C. Culmone, and P. Breedveld, "Surgical drilling of curved holes in bone-a patent review," Expert Rev. Med. Devices, vol. 16, no. 4, pp. 287-298, 2019

[7] P. C. López-Custodio, J. S. Dai, and J. M. Rico, "Branch reconfiguration of Bricard linkages based on toroids intersections: Line-symmetric case," J. Mech. Robot., vol. 10, no. 3, 2018 .

[8] K. J. Kim, "Circles in torus-torus intersections," J. Comput. Appl. Math., vol. 236, no. 9, pp. 2387-2397, 2012. 